\title{
NEWS IN BRIEF
}

\section{Mental Health Care Bill}

On August 8, the Rajya Sabha passed the new Mental Health Care Bill. It is a good step forward from the outdated Mental Health Act of 1987. One of the salient features of the new bill is that it decriminalizes the attempt to suicide. This repeals Section 309 of the Indian Penal Code which provided for a year-long imprisonment for a failed suicide bid. It recognizes a suicide bid as an extreme step of a person under unimaginable stress and attempts to help the individual instead of treating him as a criminal. Unmodified Electroconvulsive therapy (ECT) has been banned and ECT to minors can be given only after approval from the Mental Health Review Board. Such a ban would stop the ECT from being administered at small and remote places, where anesthetic support is not available.

The Bill ensures right of every person to access affordable and good quality mental health services which are to be made available by the Government. The mental health services are to be integrated into general health services, and are to be made available in each district. The Bill mandates establishment of facilities such as half-way homes, sheltered accommodations, supported accommodations, rehabilitation establishments and services, services to support family, and child and old-age mental health services.

India currently has one psychiatrist for every 400000 population, and only $1 \%$ of the health budget is spent on mental health problems. This new Bill may show the right way forward to deal with the gargantuan and difficult problems of people with mental illnesses.

(The Hindu 13 August 2016)

\section{CRISPR TECHNOLOGY GETS FDA NoD}

CRISPR technology is the gamechanger in medicine today. And now the US National Institutes of Health (NIH) has approved a proposal to use CRISPR-Cas9 in humans. This has caused a storm of excitement in the biotechnology world.

CRISPR stands for Clustered Regularly Interspaced Short Palindromic Repeats. It is a gene sequence discovered in bacteria and found to be a self defence tool against viruses. This part of the bacterial genome actually secretly annexes a part of the viral DNA when infected by a particular virus. When it encounters a similar viral DNA again, it activates the Cas9 enzyme to cut out the viral DNA from its genome.Scientists have now developed tools to activate this CRISPR sequence and use it to splice any gene of interest. Hence it is a remarkable gene editing tool at a cost $99 \%$ lower than previous techniques.

Chinese scientists will be the first in the world to use CRISPR technology in a trial on lung cancer patients. They will extract immune cells from the patients with incurable lung cancer and use CRISPR to add a new genetic sequence which will help their immune system target and destroy the cancer. The cells will then be re-introduced into the patients' bloodstream.

The trial in the US is being funded by a foundation of former Facebook president Sean Parker. The researchers will remove $\mathrm{T}$ cells from 18 patients with several types of cancers and perform three CRISPR edits on them. One edit will insert a gene for a protein which instructs the $\mathrm{T}$ cells to target cancer cells, and a second edit removes a natural Tcell protein that could interfere with this process. The third will remove the gene for a protein that prevents the cancer cells from disabling $\mathrm{T}$ cells. The researchers will then infuse the edited cells back into the patient. If CRISPR succeeds in these patients it will change the way we practice medicine forever. (Nature 22 June 2016)

\section{Should Children Play 'Pokemon Go'}

It is being dubbed "the greatest unintentional health fad ever." Suddenly all around the world, children are moving outdoors. They are socializing and exploring new places, including museums and parks. What is this new phenomenon which has taken the world by storm?

Pokemon Go; a new augmented reality mobile game, which has been downloaded by more than 100 million people worldwide. A game in which strange creatures called pokemon are superimposed on your own real world locations. And the challenge is to locate, train and capture them. As the game is necessarily played outdoors, children are moving outdoors in droves.

There have been several other unintended positive effects of this game, like autistic children are suddenly learning to move out and mix with new people. But on the flip side, there are safety issues. Children need to be careful when they start moving in unfamiliar new environments. Potential predators can either lure children to lonely spots or take notice as they visit the same locations repeatedly. Parents should consider playing the game with their kids. At the least, they need to know how the game is played and be aware of where their kids are going to play it.

(The Hindu 12 July 2016)

Gouri Rao Passi gouripassi@hotmail.com 